\title{
Generation and characterization of nano aluminium powder obtained through wire explosion process
}

\author{
T K SINDHU, R SARATHI* and S R CHAKRAVARTHY ${ }^{\dagger}$ \\ Department of Electrical Engineering, ${ }^{\dagger}$ Department of Aerospace Engineering, Indian Institute of Technology \\ Madras, Chennai 600 036, India
}

MS received 2 January 2007; revised 8 March 2007

\begin{abstract}
In the present study, nano aluminium particles were produced by wire explosion process (WEP) in nitrogen, argon and helium atmospheres. Thus produced nano particles were characterized through certain physico-chemical diagnostic studies using wide angle $\mathrm{X}$-ray diffraction (WAXD) and by energy dispersive analysis by X-rays (EDAX). The size and shape of the powder were analysed by using transmission electron microscopic (TEM) studies. The particle size distribution studies were performed by adopting log-normal probability distribution. The relationship between size of the particle generated in the explosion process and the type of inert gas/pressure was analysed. The mechanisms of nano particle formation, the factors which can aid the process of formation of nano particle in the wire explosion process were analysed. It is realized that energy deposited to the conductor and duration of current flow have major impact on particles produced by this process.
\end{abstract}

Keywords. Nano particles; wire explosion process; aluminium particle; log-normal distribution; WAXD; EDAX; TEM.

\section{Introduction}

Nanoparticles are particles of size $<100 \mathrm{~nm}$ in diameter that exhibit enhanced size-dependent properties compared to ultra-fine or larger particles of the same material (Gleitzer 2000). Aluminium powders are common ingredients for explosive formulations. It has been shown that the use of nano-aluminium increases the propellant burning rate further, and gives rise to sub-micron aluminium oxide as a combustion product. This could be useful in reduced smoke applications, since the visibility of the smoke varies as the square of the product-oxide size. Thus, the use of aluminium at the nano-particle size level enables reduced smoke while still ensuring the high specific impulse offered by aluminized propellants (Ivanov et al 2003; De Luca et al 2005).

Further, the use of nano-aluminium can also mitigate two-phase losses to thrust, and in judicious combination with regular aluminium, may lead to optimal control of combustion instability due to appropriate particulate damping. A low concentration of regular-sized aluminium would also obviate agglomerate formation, which is a major impediment in deciding the appropriate size of the regular aluminium in conjunction with the other propellant formulation variables (De Luca et al 2005).

Generation of ultra fine metal particles is difficult to obtain by mechanical methods. Nano particle formation by

*Author for correspondence (rsarathi@iitm.ac.in) wire explosion technique is basically an inert gas evaporation technique, where the particles are produced by evaporating a thin metal conductor by passing high current to it, in an inert atmosphere (Hahn and Averback 1990; Jiang and Yatsui 1998; Rhee et al 2002). This approach is basically a top-down approach to produce nano metal powders.

Having known all this, in the present study, the nano aluminium particles were produced by wire explosion process (WEP) in nitrogen, argon and helium atmospheres and characterized through certain physico-chemical diagnostic studies using wide angle X-ray diffraction (WAXD) and energy dispersive analysis by X-rays (EDAX). The size and shape of the powder were analysed by using transmission electron microscopic (TEM) studies. The particle size distribution studies were performed by adopting log-normal probability distribution. The relationship between the size of the particle generated in the explosion process and the type of inert gas/pressure was analysed. The suitable atmospheres for producing pure nano size aluminium particle were identified. The mechanism of nano particle formation and the factors which can aid the process of formation of nano particle in the wire explosion process were analysed.

\section{Experimental}

The factors which can influence the particle size produced by the wire explosion process are (i) electrical circuit parameters, (ii) the operating pressure of the gas in the 
wire exploding chamber, (iii) amount of energy deposited onto the exploding conductor, (iv) the diameter and length of the exploding conductor, (v) melting point of the exploding conductor, (vi) thermal conductivity of the medium and (vii) breakdown strength of the medium.

\subsection{Identification of circuit parameters of wire exploding unit}

The basic circuit used for exploding the wires to form nano powders is shown in figure 1. The capacitor is charged from the source and discharged through the wire. The capacitor is charged through voltage doubler circuit. Energy stored in the capacitor, $W=0.5 \mathrm{CV}^{2}$, where $C$ is the capacitance value and $V$ the charged voltage of the capacitor. By varying the charging voltage, it is possible to deposit required energy. By closing of switch, $S$ (in figure 1), the voltage appears across the wire and the current (controlled by the RLC circuit) rises, causing joule heating of the conductor, which eventually melts. The switch, $S$, is a high voltage trigatron gap, $R$ the exploding wire resistance and $L$ the contribution by the internal inductance of the capacitor and the lead inductance. The basic circuit works like an RLC circuit and maximum power it could deliver is VI. The typical voltage across $R$ and current flow through it, were measured at the time of explosion, using the voltage probe (EP-50k, PEEC. A Japan) and current probe (Pearson Electronics, USA, Current transformer Model No-101), and is shown in figure 2. The magnitude of current flow in the circuit depends on the resistance and the inductance in the circuit (Hayt et al 2002). In the present work, the circuit parameters match with the condition for the RLC under-damped circuit, where

$$
\frac{R^{2}}{4 L^{2}}<\frac{1}{L C}
$$

Then the magnitude of current flow in the circuit can be written as

$$
i(t)=\frac{E e^{\frac{-R}{2 L} t}}{\sqrt{\frac{L}{C}-\frac{R^{2}}{4}}} \sin \left(\sqrt{\frac{1}{L C}-\frac{R^{2}}{4 L^{2}}} t\right) .
$$

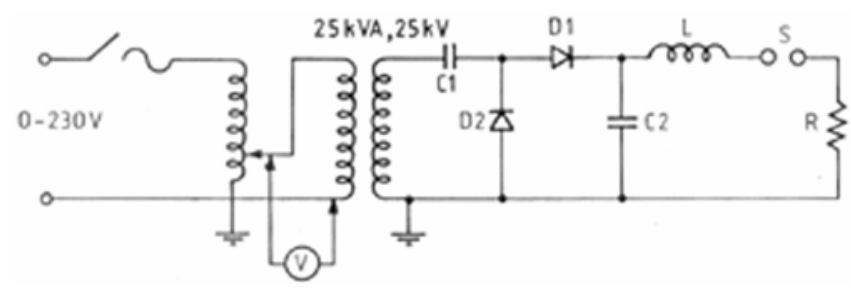

Figure 1. Experimental set up.
This suggests a choice of the capacitor with low $L / C$ ratio. In (2) above, if the exponential term is absent, the current waveform looks like a perfect sinusoidal current. Also, to have short rise time for the current, the inductance in the circuit should be as minimum as possible. Sedoi et al (1999) mentioned that the efficiency of wire explosion process is high with high $\mathrm{d} i / \mathrm{d} t$ with the magnitude of current flow of $10^{11} \mathrm{~A} / \mathrm{m}^{2}$. The circuit parameters of the experimental set up used in the present study is provided in table 1 .

\subsection{Factors which influence nano particle formation in wire explosion process}

The major factor determining the particle size in the wire explosion process is the evaporation rate of the exploding conductor and the condensation mechanism of the evaporated conductor. The factors which can alter the process of evaporation are (i) current density and (ii) magnetic pressure developed due to current flow (z-Pinch).
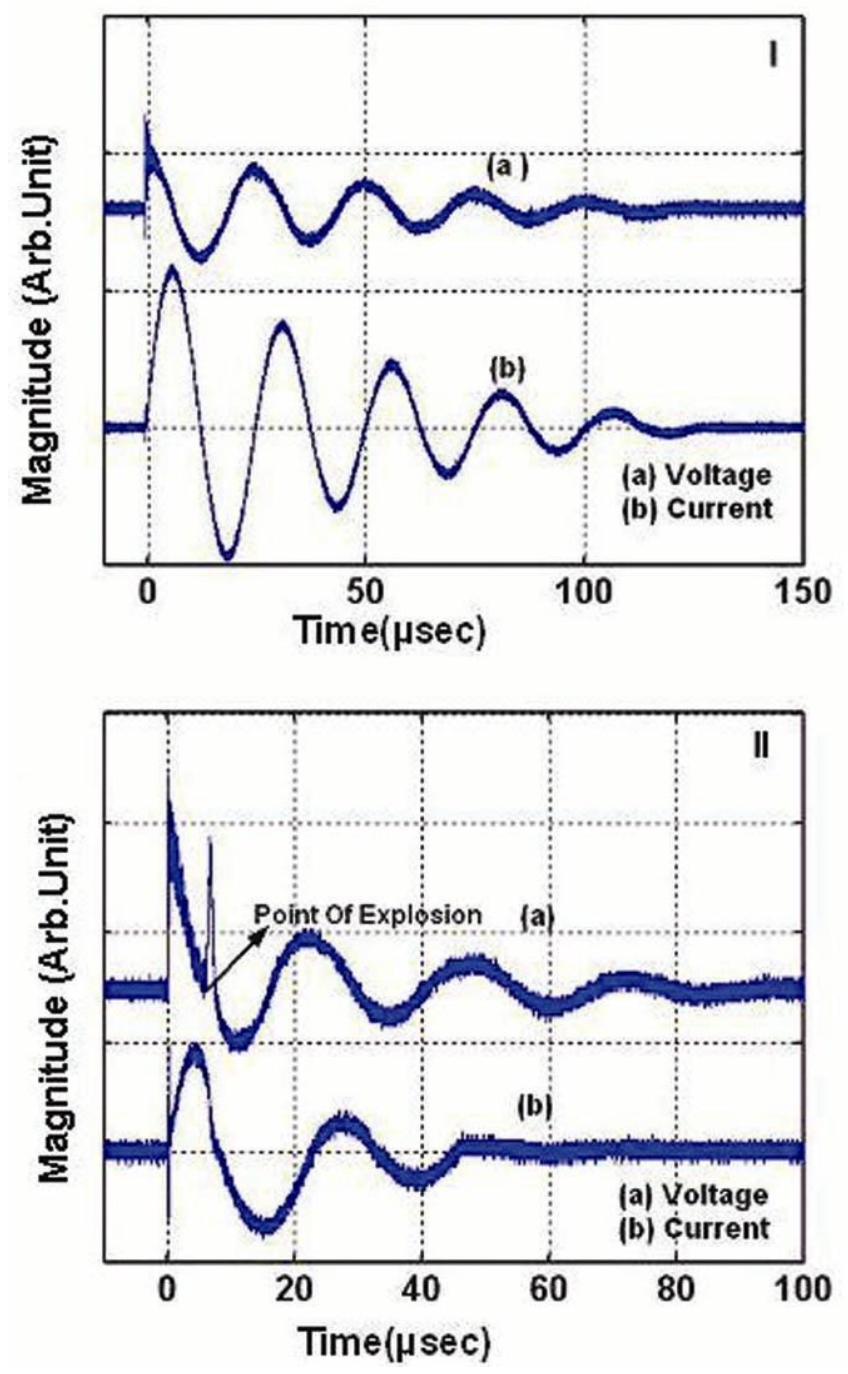

Figure 2. Typical voltage and current waveforms: (I) without explosion and (II) with explosion. 
2.2a Characteristic variation of current density in the exploding conductor: The conduction current density at any point within the conductor can be written as (Hayt and Buck 2005)

$$
j_{\mathrm{z}}(r)=j_{0} e^{-r \sqrt{\Pi f \mu \sigma}}
$$

where $j_{0}$ is the current density at the surface, $f$ the frequency of current signal, $\mu$ and $\sigma$ are the permeability and conductivity of the conducting path. The typical variation in current density in the conductor due to injection of current pulse to the conductor, with time is shown in figure 3 . It is realized that the current density is uniform along the axis of the conductor. Current density, $j$, has to satisfy the basic ampere's circuital law, $j=\mu_{0}^{-1} \nabla \times B$ and the magnetic field developed in the process due to the current flow should satisfy the basic condition $\nabla \cdot B=0$ (Hayt and Buck 2005). In general, it is essential to have the ratio between the gas kinetic pressure and magnetic pressure in the fluid volume to be minimum for having uniform current density in the exploding conductor.

$2.2 \mathrm{~b}$ Characteristic variation in resistivity of exploding conductor: By discharging high current through the wire, due to Joule heating, the temperature of the conductor rises and heating of liquid phase takes place, followed by melting and eventually reaches the boiling point and superheating, before the liquid phase changes to gas phase. At the

Table 1. Summary of experimental set up.

\begin{tabular}{ll}
\hline Capacitance & $3 \mu \mathrm{F}$ \\
Charging voltage & $25 \mathrm{kV}$ \\
Material & Aluminium \\
Wire diameter & $0.5 \mathrm{~mm}, 0.25 \mathrm{~mm}$ \\
Length of the wire & $140 \mathrm{~mm}$ \\
Ambience & Nitrogen, argon and helium \\
\hline
\end{tabular}

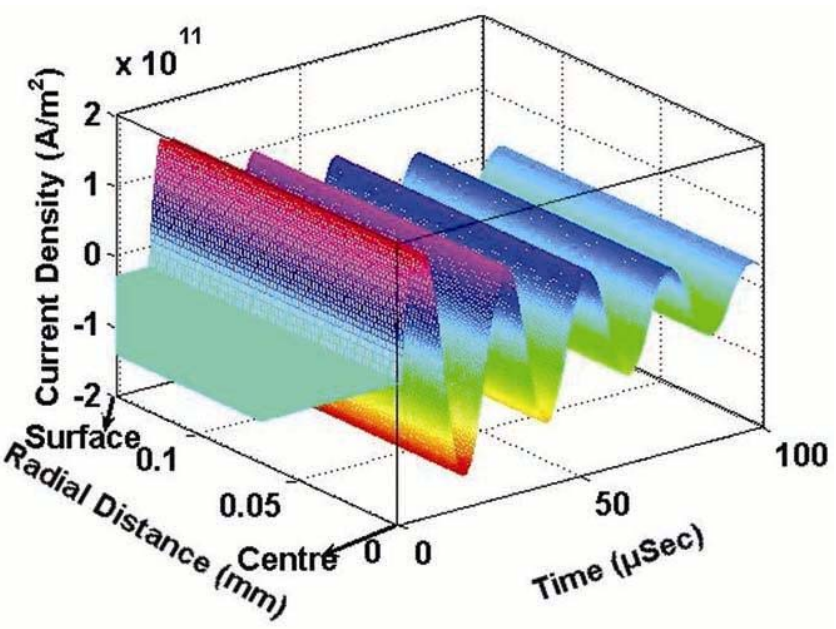

Figure 3. Typical variations in current density in the exploding conductor due to injection of current pulse. heating stage, the material resistance is determined by the introduced energy.

For calculation of resistance of the wire, the temperature $(T)$, density $(\gamma)$ and dependence of resistivity $(\rho)$ are approximated as

$$
\rho(\gamma, T)=\rho_{0}\left[1+\beta\left(T-T_{0}\right)\right]\left(\frac{\gamma}{\gamma_{0}}\right),
$$

where $\gamma=\gamma_{0}\left[1-\alpha(T)\left(T-T_{0}\right)\right], \quad \gamma_{0}$ is the density at the melting temperature, $\beta$ the temperature coefficient of resistivity and $\alpha$ the thermal expansion coefficient (Kuskova et al 1997). From this it could be realized that the wire resistance is altered during the evaporation process.

2.2c Influence of magnetic pressure developed due to current flow in the exploding conductor: In general, when a pulsative current of magnitude, $I$, is applied to the conductor with radius $(a)$, the magnetic pressure $(P)$ developed at any point $(r)$ in the zone is given by (Godbloed and Poedts 2004)

$$
P=P_{\mathrm{c}}\left(1-\frac{r^{2}}{a^{2}}\right),
$$

where the central magnetic pressure $\left(P_{\mathrm{c}}\right)$ is related to current flow through the conductor by

$$
P_{\mathrm{c}}=\frac{\mu_{0} I^{2}}{4 \pi^{2} a^{2}} .
$$

Figure 4 shows the characteristic variation in the magnetic pressure developed due to uniform current flow in the system. It is realized that the magnetic pressure is maximum at the centre and is minimum near the surface of the conductor and hence a surface layer evaporates and plasma is formed before explosion. The plasma zone shunts the

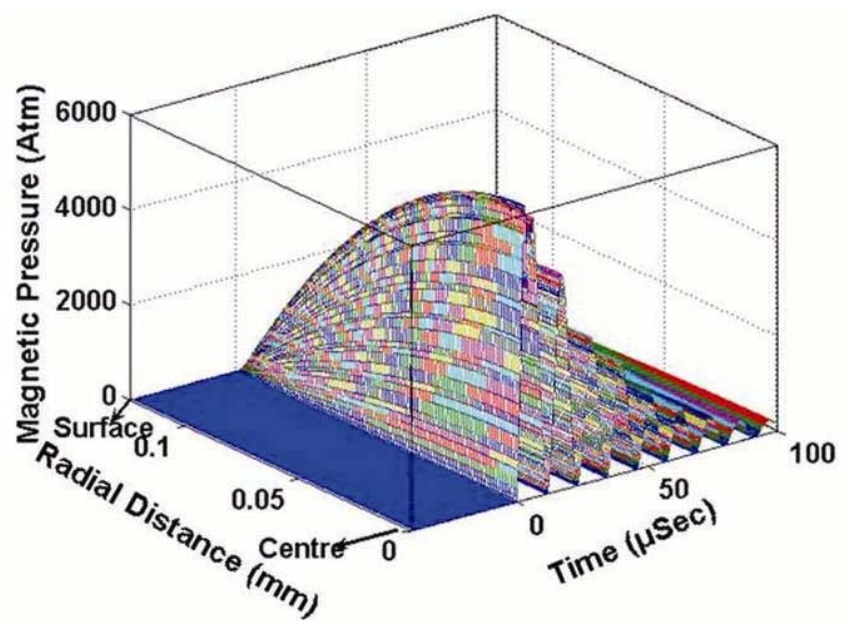

Figure 4. Characteristic variation in the magnetic pressure developed due to current flow in exploding conductor. 
current flowing through the conductor. Conduction in the conductor continues until the vapours are optically thick and radiation is emitted from the surface. At this stage, a column of nearby or completely ionized plasma is formed leading to magneto hydrodynamic instability. The time required for MHD instabilities to occur is

$$
t_{\mathrm{MHD}} \approx 6 \frac{\pi^{2} a^{2}}{I}\left(\frac{\rho}{\mu_{0}}\right)^{0.5},
$$

where $a$ is the radius of the conductor, $\mu_{0}$ the permeability and $\rho$ the density (Godbloed and Poedts 2004). Hence it is clear that inner core continues to carry current till the point of occurrence of MHD instabilities by the explosion process.

\subsection{Physico-chemical diagnostic studies}

The WAXD measurements were done using Phillips X-ray diffractometer. A scan rate of $2 \% / \mathrm{min}$ at 2000 cycles using $\mathrm{CuK} \alpha$ radiation of wavelength, $1.5426 \AA$, was applied. Compositional analysis of the produced nano particles was carried out by energy dispersive analysis of X-ray (EDAX). The particle size measurements were carried out through TEM studies (CM-12 scanning transmission electron microscopy with EDAX attached). The particle size was evaluated based on TEM bright field images. The number of analysed particles for each synthesis was around 500 .

\section{Results and discussion}

The particle size produced by the wire explosion process reduces substantially with increasing super heating of the metal by injecting excess energy to the conductor, i.e., $k=W / W_{\text {s }}$, where $W$ is the energy injected into the evaporating wire and $W_{\mathrm{s}}$ the sublimation energy of the wire.
The sublimation energy of aluminium is $33 \mathrm{~J} / \mathrm{mm}^{3}$ (Chandler et al 2002). In the present work, experiments were carried out by injecting different levels of energy to the conductor. The diameter of the conductor used for understanding the impact of energy deposition is $0.25 \mathrm{~mm}$ diameter aluminium conductor. Figure 5 shows the voltage and current waveforms measured at different levels of deposition of energy. During evaporation of metal conductor the conductivity of the conductor drastically reduces resulting in current cut-off in the circuit and the voltage overshoots across the circuit inductor, $L$, whose amplitude is $V_{\mathrm{L}}=L \mathrm{~d} i / \mathrm{d} t$.

In the wire explosion process, the energy deposited to the exploding conductor could be calculated from the measurement of voltage and current wave shapes. It is possible to relate the energy deposited to the conductor and the maximum temperature attained by the conductor by the fundamental equation

$$
Q=C_{\mathrm{s}} * \Delta T_{\mathrm{m}}+h_{\mathrm{m}}+C_{\mathrm{f}} * \Delta T,
$$

where $Q$ is the energy deposited, $C_{\mathrm{s}}$ the specific heat capacity of the aluminium conductor, $\Delta T_{\mathrm{m}}=T_{\mathrm{m}}-T_{0}$, where $T_{\mathrm{m}}$ is the melting temperature and $T_{0}$ the initial temperature, $h_{\mathrm{m}}$ the melting heat, $C_{\mathrm{f}}$ the heat capacity of the liquid metal, $\Delta T=T-T_{\mathrm{m}}$, where $T$ is the final temperature, and $T_{\mathrm{m}}$ the melting temperature (Tkachenko et al 2004). It is clear from the magnetic pressure plot (figure 4) that the magnetic pressure exerted on the surface layer is zero. So the surface layer will evaporate after the boiling point is crossed and the remaining inner layers will continue to carry current till the point of MHD instabilities. In this process, before application of the high current through the exploding wire, the temperature of the conductor was at atmospheric temperature.

After wire evaporation, the plasma begins to expand in the medium due to the enormous difference in the temperature and pressure between the plasma and the ambient
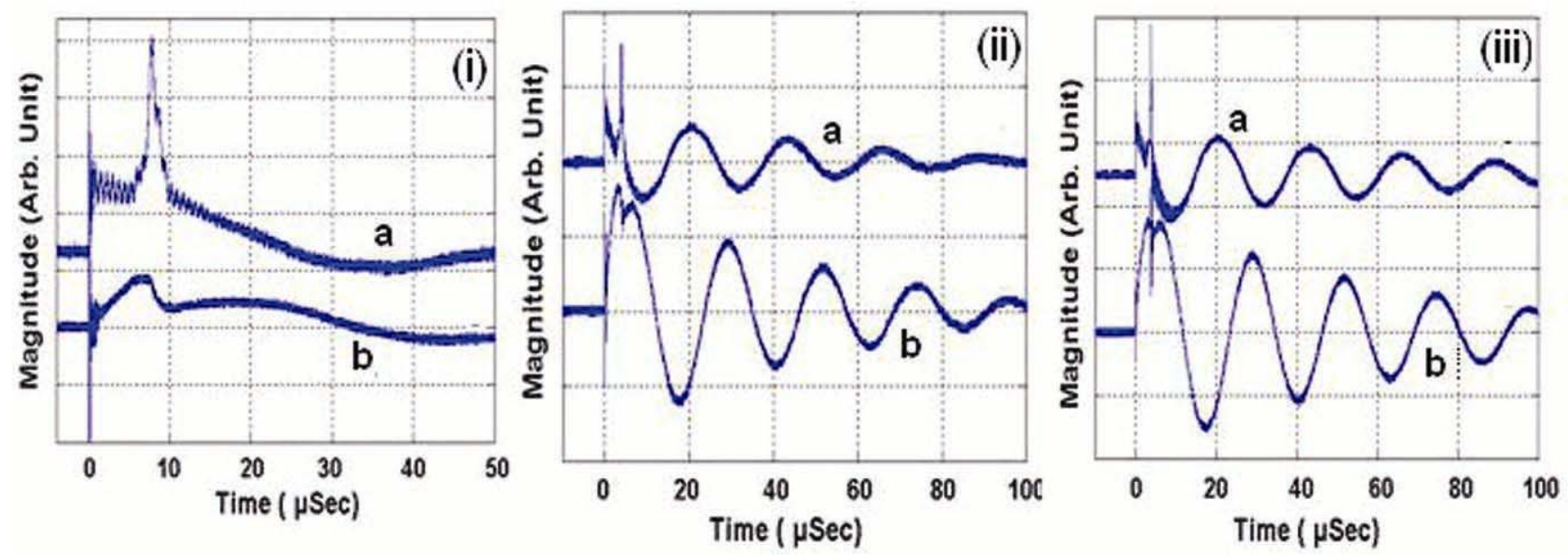

Figure 5. Voltage and current waveforms for different injected energies: (i) w/ws $=1$, (ii) $\mathrm{w} / \mathrm{ws}=2$, (iii) $\mathrm{w} / \mathrm{ws}=3$. (a) Voltage and (b) current. 
Table 2. Characteristic variation in process parameters in WEP at different ambience.

\begin{tabular}{llcll}
\hline Atmosphere & $k$ & $\tau(\mu \mathrm{s})$ & $T_{\mathrm{MHD}}(\mu \mathrm{s})$ & $T^{\prime}\left({ }^{\circ} \mathrm{C}\right)$ \\
\hline Argon (diameter of & 1 & $4 \cdot 55 \pm 0 \cdot 193$ & $4 \cdot 74$ & $4500 \pm 100$ \\
$\quad$ the conductor is & 2 & $3 \cdot 36 \pm 0 \cdot 182$ & $3 \cdot 16$ & $5950 \pm 130$ \\
$0.25 \mathrm{~mm})$ & 3 & $2 \cdot 95 \pm 0.241$ & $2 \cdot 52$ & $6782 \pm 70$ \\
Argon* & 1 & $6 \cdot 83 \pm 0 \cdot 362$ & $7 \cdot 1$ & $4325 \pm 100$ \\
Helium* & 1 & $6 \cdot 85 \pm 0.317$ & $6 \cdot 9$ & $4250 \pm 200$ \\
Nitrogen* & 1 & $5 \cdot 99 \pm 0.203$ & $6 \cdot 2$ & $4380 \pm 150$ \\
\hline
\end{tabular}

*Wire diameter of $0.5 \mathrm{~mm}$ used for the study; $T^{\prime}$, temperature at the time of explosion; $T$, time of explosion; $T_{\mathrm{MHD}}$, time of magneto hydrodynamic instability

gas. The expanded plasma particles are gradually cooled during this process of collision with ambient gas molecules. Finally, the plasma loses its expansive driving force. Hence, a low ambient pressure allows a large expansion volume. This leads to low number concentration of the substance during particle growth, resulting in nano-particles.

Table 2 shows the characteristic variation in time of explosion, time of MHD instability occurrence and the maximum temperature attained by the conductor. It is realized that higher the level of energy injected to the conductor, lower the time of explosion. This indicates that higher $\mathrm{d} i / \mathrm{d} t$ allows the temperature of the conductor to reach the supersaturated state leading to discontinuity in current flow. The temperature of the conductor calculated by (8) is provided in table 2 . This indicates that higher energy deposition increases the temperature of the conductor. It is noticed that increase in diameter of the conductor shows increase in time of explosion and occurrence of MHD instability. Also the experimental time of explosion and calculated time for MHD instability to occur matches nearly indicating that injection of energy to the conductor continues until MHD instability occurs.

Figure 6 shows WAXD spectra of the nano aluminium powder obtained through the wire explosion technique by injecting different levels of energy equivalent/more than the sublimation energy of the material, in the argon atmosphere. It is realized through WAXD studies, the aluminium powder obtained through wire explosion process, in the argon ambience, by injecting different levels of energy (figures 6a-c), did not show any oxide formation.

The WAXD of nano aluminium powder obtained through wire explosion process in helium and nitrogen atmospheres is also shown in figures $6 \mathrm{~d}$ and e. The characteristic of nano aluminium powder obtained in helium ambience (figure 6d) is much similar to the powder obtained in argon ambience. It is realized that certain additional peaks were seen for the aluminium powder generated in the nitrogen atmosphere (figure 6e), which are characteristic peaks of hexagonal aluminium nitride. This gives an indication that, the pure aluminium particles could be generated in the helium and argon ambience.

The composition of the aluminium powder produced by the wire explosion process was determined by energy dispersive analysis by X-rays. The typical EDAX pattern

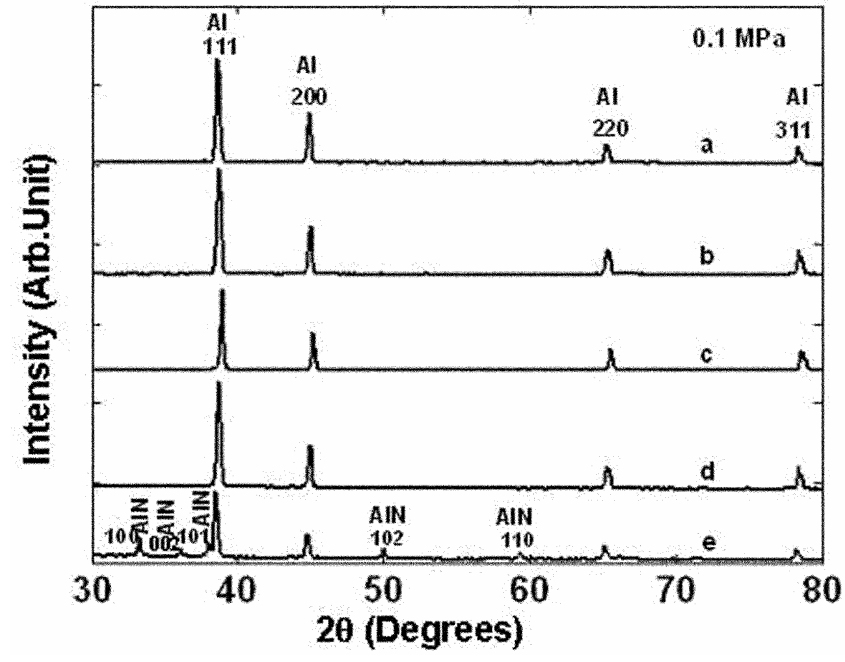

Figure 6. WAXD pattern of nano aluminium powder: a $\mathrm{w} / \mathrm{ws}=1, \mathrm{~b} . \mathrm{w} / \mathrm{ws}=2, \mathrm{c} . \mathrm{w} / \mathrm{ws}=3$ (in $\operatorname{argon}$ ), d. helium and e. nitrogen.

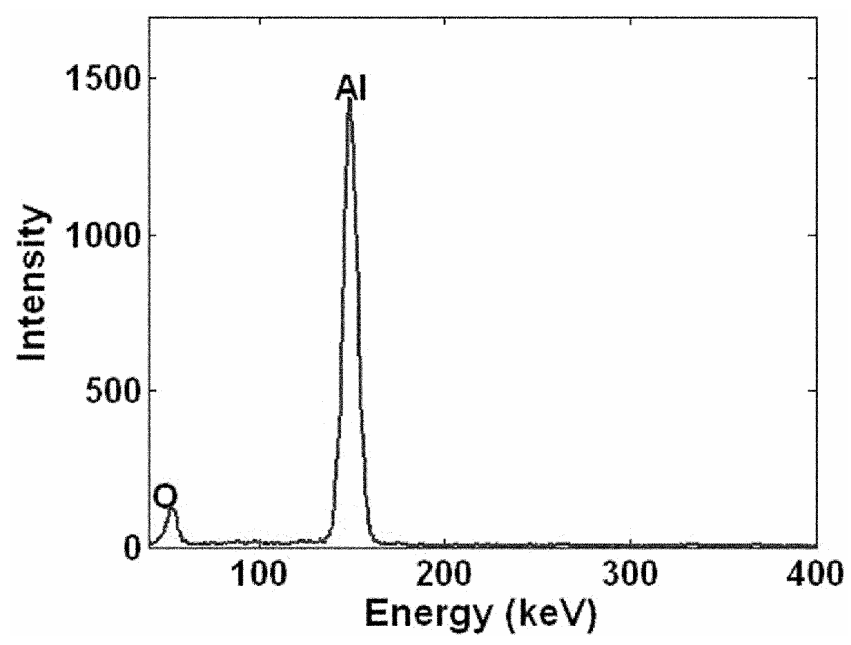

Figure 7. Typical EDAX pattern of the nano aluminium particle produced by wire explosion process

obtained for the aluminium particle produced by wire explosion process is shown in figure 7. The compositional analyses of the nano aluminium particles obtained in different ambience are shown in table 3 . It is realized 
that the weight percentage of aluminium is high with the particles produced in helium and argon atmospheres. The results of the study indicate that the aluminium particles produced in the nitrogen medium shows presence of nitro-
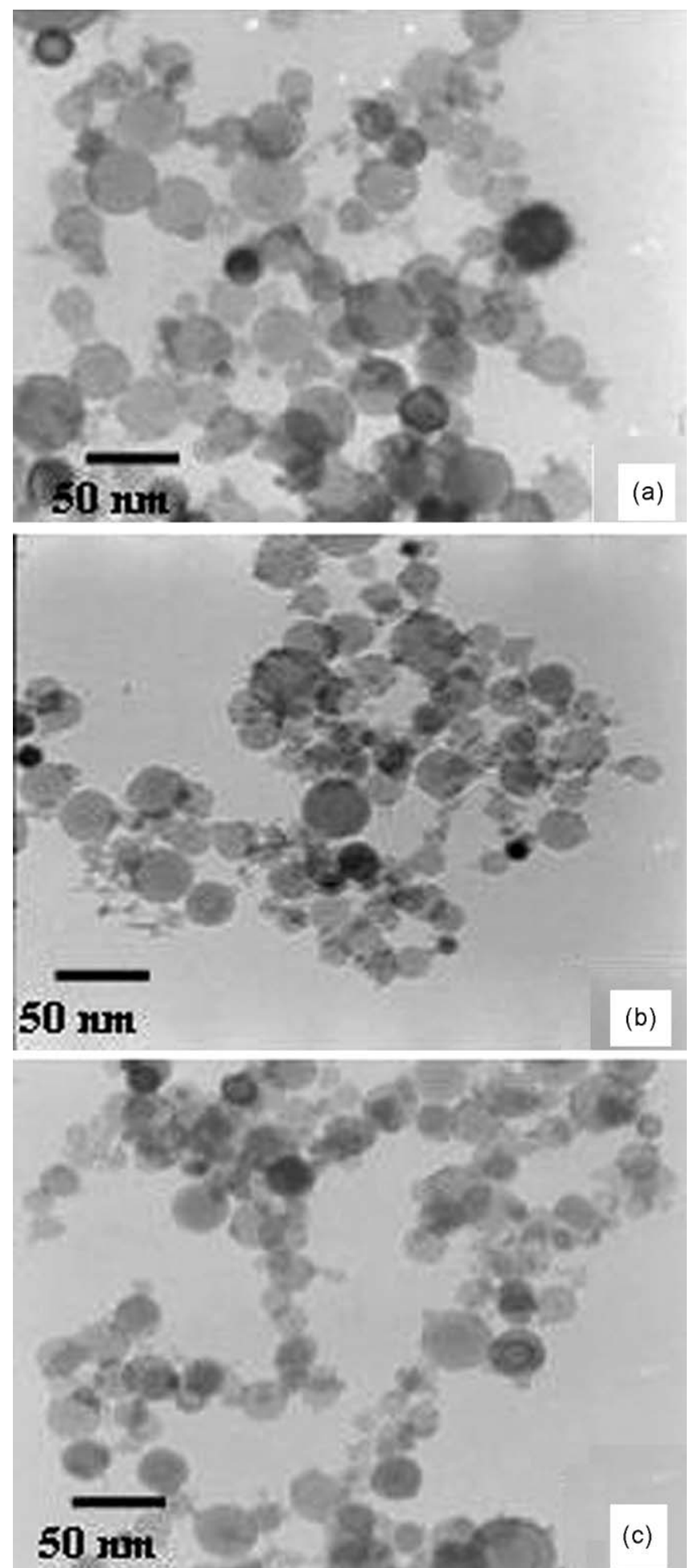

Figure 8. TEM pictures of the nano aluminium particles obtained in argon atmosphere: (a) w/ws $=1,(\mathbf{b}) \mathrm{w} / \mathrm{ws}=2$ and (c) $\mathrm{w} / \mathrm{ws}=3$. gen content. Also the weight percentage of aluminium content in it is less. It is observed that the major composition of the powder produced by the explosion process is aluminium and oxygen for the particles produced in the argon/helium atmosphere.

The percentage of aluminium on the produced particle through wire explosion process for different levels of energy deposited to the exploding conductor is provided in table 4 . It is realized that the percentage of oxygen content is different for the levels of energy deposited. Comparing the size of particles produced for different levels of energy deposited and particle size produced, it is realized that there is an optimum level of energy to be deposited for producing high percentage of aluminium particle. If the energy deposition is high the size of the produced particle may be low and they are prone for oxidation, which could be realized from the EDAX results.

Figure 8 shows the TEM pictures of the particles produced by the wire explosion process by injecting different levels of energy to the exploding wire in the argon atmosphere. The particles obtained in the argon medium are of spherical shape with $f c c$ structure of the aluminium particle. Figure 9 shows the TEM structure of nano aluminium particles obtained by the wire explosion process at different ambience. The particles produced in nitrogen show near

Table 3. EDAX analysis of nano aluminium powder.

\begin{tabular}{|c|c|c|c|}
\hline Gas & Pressure (MPa) & Element & Weight (\%) \\
\hline Argon* & $\begin{array}{l}0.1 \\
0.05\end{array}$ & $\begin{array}{c}\mathrm{O} \\
\mathrm{Al} \\
\mathrm{O} \\
\mathrm{Al}\end{array}$ & $\begin{array}{r}5 \cdot 50 \\
94 \cdot 50 \\
10 \cdot 70 \\
89 \cdot 30\end{array}$ \\
\hline Helium* & $\begin{array}{l}0.1 \\
0.05\end{array}$ & $\begin{array}{l}\mathrm{O} \\
\mathrm{Al} \\
\mathrm{O} \\
\mathrm{Al}\end{array}$ & $\begin{array}{c}5 \cdot 40 \\
94 \cdot 6 \\
3 \cdot 70 \\
96 \cdot 3\end{array}$ \\
\hline Nitrogen* & 0.05 & $\begin{array}{l}\mathrm{O} \\
\mathrm{N} \\
\mathrm{Al} \\
\mathrm{O} \\
\mathrm{N} \\
\mathrm{Al}\end{array}$ & $\begin{array}{c}22 \cdot 3 \\
3 \cdot 30 \\
74 \cdot 5 \\
31 \cdot 1 \\
1 \cdot 60 \\
67 \cdot 2\end{array}$ \\
\hline
\end{tabular}

*Diameter of the conductor is $0.5 \mathrm{~mm}$

Table 4. EDAX analysis of nano aluminium powder produced at different energy levels.

\begin{tabular}{ccc}
\hline$k^{*}$ & Element & Weight (\%) \\
\hline 1 & $\mathrm{~A} 1$ & 94.5 \\
& $\mathrm{O}$ & 5.50 \\
2 & $\mathrm{Al}$ & 96.5 \\
& $\mathrm{O}$ & 3.50 \\
3 & $\mathrm{Al}$ & 94.8 \\
& $\mathrm{O}$ & $5 \cdot 20$ \\
\hline
\end{tabular}

*Diameter of the conductor is $0.25 \mathrm{~mm}$ 

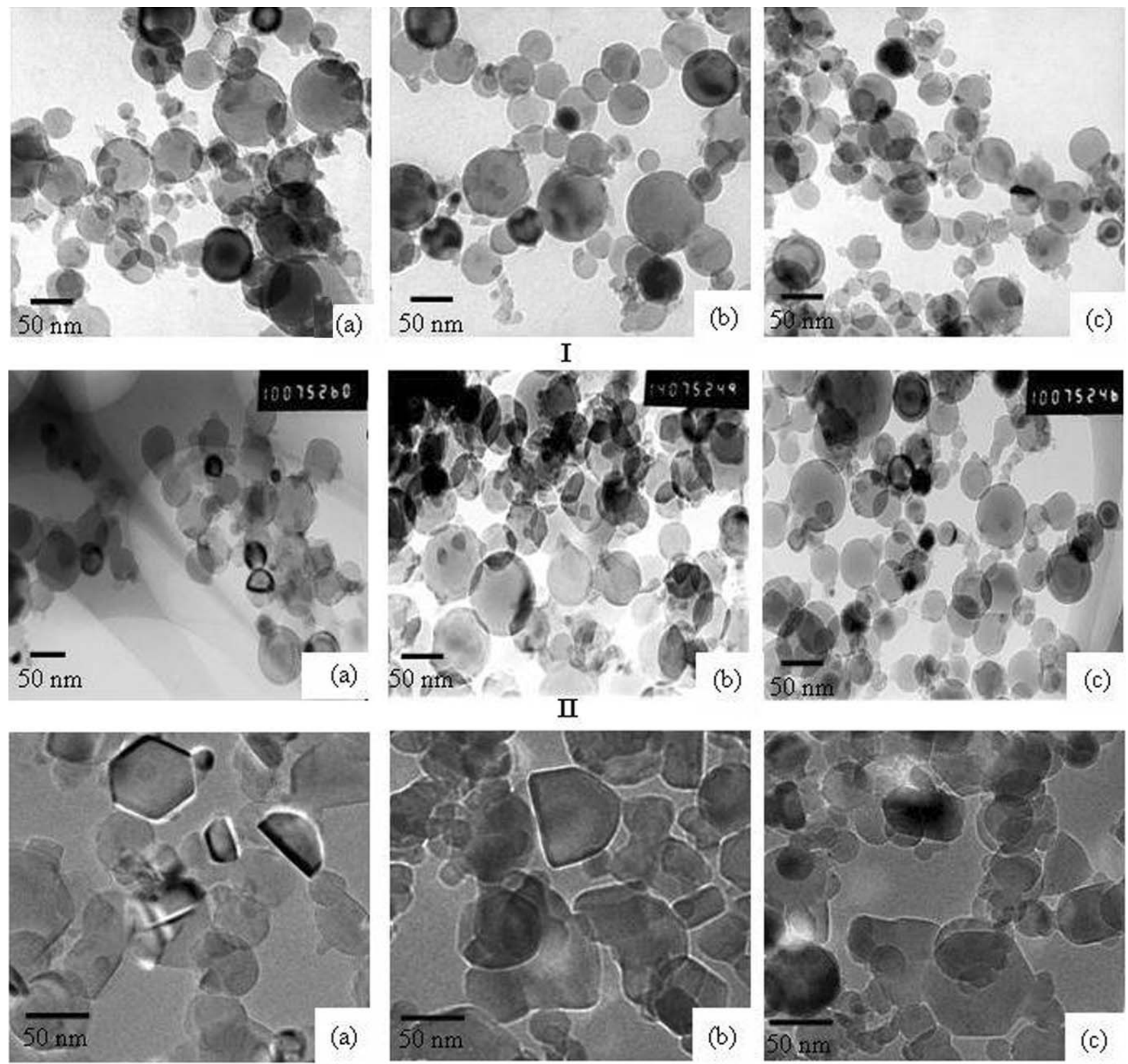

III

Figure 9. TEM of nano aluminium particle obtained by the wire explosion process at different ambience: (I) helium, (II) argon and (III) nitrogen. (a) $0.025 \mathrm{MPa}$, (b) $0.05 \mathrm{MPa}$ and (c) $0.1 \mathrm{MPa}$.

circular shape with corrugated edge and some particles are of hexagonal in shape.

The particle size lies in the range of a few $\mathrm{nm}$ to $100 \mathrm{~nm}$. It is observed that the particle size measurement follows the log-normal distribution (Claus Bernhardt 1994).

$$
f(d)=\frac{1}{\sqrt{2 \pi} d \log \sigma_{\mathrm{g}}} \exp \left\{-\frac{\left(\log d-\log D_{50}\right)^{2}}{2\left(\log \sigma_{\mathrm{g}}\right)^{2}}\right\},
$$

$$
\begin{aligned}
\log \sigma_{\mathrm{g}} & =\sqrt{\frac{\sum n_{i}\left(\log d_{i}-\log D_{50}\right)^{2}}{\sum n_{i}},} \\
\log D_{50} & =\frac{\sum n_{i} \log d_{i}}{\sum n_{i}} .
\end{aligned}
$$

Here $f(d)$ denotes the log-normal distribution, $d$ the particle diameter, $n_{i}$ the number of particles with diameter $d_{i}, D_{50}$ 

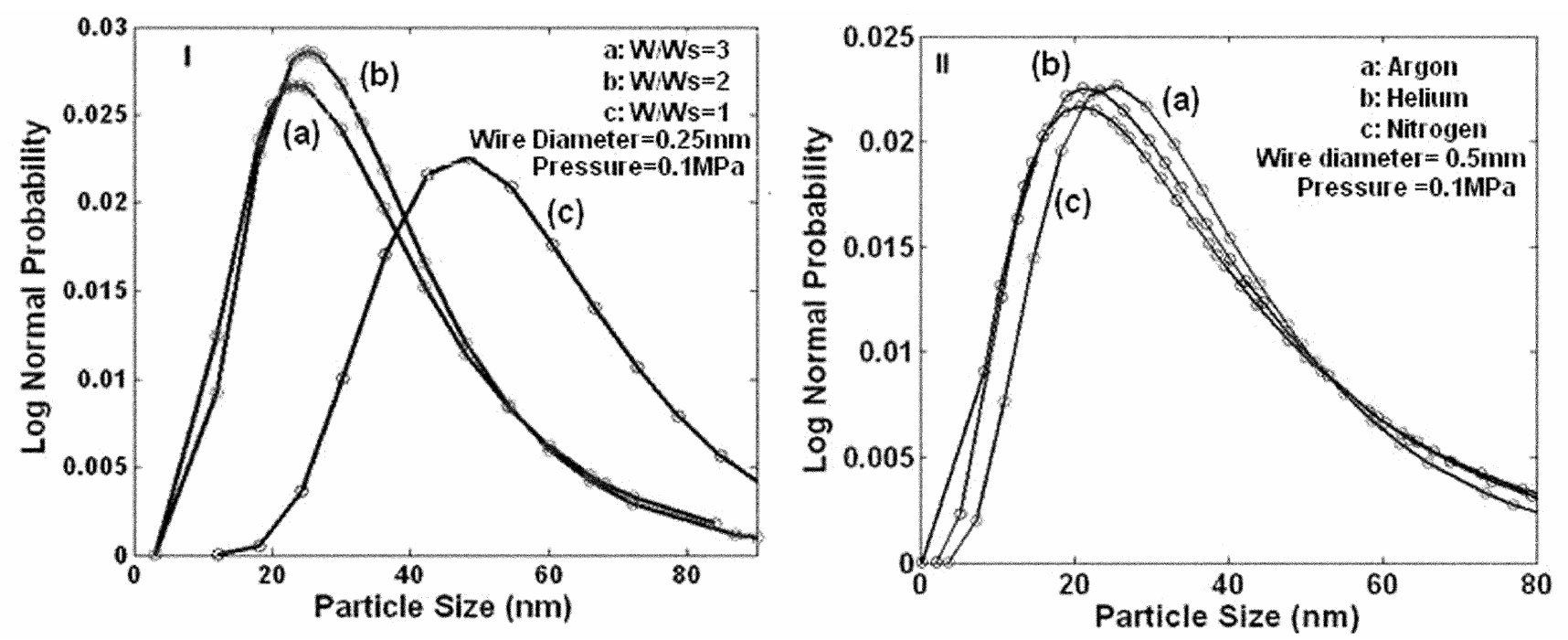

Figure 10. Particle size distribution of nano aluminium powder: (I) different levels of energy injected and (II) different atmospheric conditions.

Table 5. Median diameter and geometric standard deviation of particles produced in different ambience at different pressures.

\begin{tabular}{lccc}
\hline Ambience & $\begin{array}{c}\text { Pressure } \\
(\mathrm{MPa})\end{array}$ & $\begin{array}{c}\text { Median dia- } \\
\text { meter, } D_{50}(\mathrm{~nm})\end{array}$ & $\begin{array}{c}\text { Geometric } \\
\text { standard deviation }\end{array}$ \\
\hline Helium & 0.025 & 36.19 & 1.92 \\
& 0.05 & 35.40 & 1.97 \\
Argon & 0.1 & 33.30 & 1.93 \\
& 0.025 & 32.00 & 1.80 \\
& 0.05 & 37.00 & 1.60 \\
& $0 \cdot 1$ & 41.00 & 1.65 \\
& $0 \cdot 1^{*}(k=1)$ & 53.41 & 1.42 \\
& $0 \cdot 1^{*}(k=2)$ & 32.00 & 1.63 \\
& $0 \cdot 1^{*}(k=3)$ & 31.74 & 1.72 \\
& 0.025 & 48.00 & 1.74 \\
& 0.05 & 36.75 & 2.00 \\
& 0.1 & 33.70 & 1.80 \\
\hline
\end{tabular}

*Diameter of the exploding wire is $0.25 \mathrm{~mm}$

the median diameter, and $\sigma_{\mathrm{g}}$ the geometrical standard deviation, respectively.

Figure 10 shows the particle size distribution of the nano aluminium particles obtained at different ambience. From figure 10(ii), it could be realized that atmospheric condition plays a major role on the particles produced. Table 5 shows the variation in median diameter and geometric standard deviation of the produced particles at different ambience. The median diameter of the particle produced at different ambience lies in the range 30$50 \mathrm{~nm}$. It is observed that increase in pressure of the ambient gas increases the size of the particle formed in the argon gas ambience. The phenomenon is different when the particles are produced in nitrogen and helium ambience. In general, argon has low thermal conductivity providing a relatively narrow arc with high current density and good electrical conductivity. Hence, at high pressure narrow arc formation with increased injected energy to the medium for long duration, the local temperature is maintained allowing nano particles formed to coagulate and sinter to form large size particles at higher pressures. It is realized that only in helium atmosphere, the change in particle size at different pressures is less. In argon and nitrogen ambience the change in size of the particle is considerable. About $22 \%$ change is noticed with argon and $31 \%$ change with nitrogen indicating that ambience plays a major role on particle formation. Also it is noticed that, the value of geometrical standard deviation of the nano aluminium powder produced, at different pressure levels, lies in the range $1.5-2$, indicating that the nucleation process is very much confined, leading to the formation of uniform-sized particles.

From particle size distribution studies, it could be realized (from figure 10(i)) that increase in injected energy to the conductor shows reduction in size of the nano particles formed. Also it is realized that increase in injected energy shows the local temperature increase of the conductor with a reduction in time of explosion of the conductor. This indicates that rate of increase in magnitude of current is important than the duration of the current flow through the conductor. With higher $w / w_{\mathrm{s}}$ ratios, after explosion process (discontinuity of solid metal exploding conductor), current starts flowing through the plasma formed zone maintaining the local temperature and allowing some of the nucleated particle in the zone to grow further causing increase in scatter of the size of particles formed.

\section{Conclusions}

It is realized that pure nano aluminium particles could be produced by wire explosion process. It is realized from WAXD studies that particles produced in nitrogen ambience show traces of aluminium nitride. It is observed that 
particles produced in helium atmosphere shows lower particle size compared to nitrogen and argon atmospheres. It could be ascertained that current density and duration of current flow play a major role on particle size formation. The shape and duration of current flow alters depending upon the level of energy deposited to the conductor. From the EDAX analysis it is realized that injecting high/low energy to the exploding conductor could indirectly control the oxide content formation. Increase in current flow for long duration allows large sized particles to form. Time of explosion of conductor and occurrence of time of magneto hydrodynamic instability correlates together indicating that higher the magnitude of current flow lower the time of explosion $/ t_{\mathrm{MHD}}$.

\section{Acknowledgement}

Two of the authors (RS and SRC) would like to thank Armament Research Board, New Delhi, for funding the project on generation and characterization of nano aluminium particles for high energy rocket propellant ingredient.

\section{References}

Chandler K M, Hammer D A, Sinars D B, Pikuz S A and Shelkovenko T A 2002 IEEE Trans. Plasma Sci. 30577
Claus Bernhardt 1994 Particle size analysis (UK: Chapman \& Hall) pp 247-253

De Luca L T, Galfetti L, Severini F, Meda L, Marra G, Vorozhtsov A B, Sedoi V S and Babuk V A 2005 Combustion, Explosion and Shock Waves 41680

Gleitzer H 2000 Acta Mater. 481

Godbloed H and Poedts S 2004 Principles of magnetohydrodynamics (Cambridge: Cambridge University Press)

Hahn H and Averback R S 1990 J. Appl. Phys. 671113

Hayt W H, Kemmerly J E and Durbin S M 2002 Engineering circuit analysis (New Delhi: Tata McGraw-Hill) 6th ed.

Hayt W H and Buck J A 2005 Engineering electromagnetics (New Delhi: Tata McGraw-Hill) 7th ed.

Ivanov Y F, Osmonoliev M N, Sedoi V S, Arkhipov V A, Bondarchuk S S, Vorozhtsov A B, Korotkikh A G and Kuznetsov V T 2003 Propellants, Explosives, Pyrotechnics 28319

Jiang W and Yatsui K 1998 IEEE Trans. Plasma Sci. 26 1498

Kuskova N I, Tkachenko S I and Koyal S V 1997 J. Phys. Condens. Matter 96175

Rhee C K, Jee G H and Kim W W 2002 J. Korean Powder Metall. Inst. 9433

Sedoi V S, Mesyats G A, Oreshkin V I, Valevich V V and Lyudmila I Chemezova 1999 IEEE Trans. Plasma Sci. 27 845

Tkachenko S I, Vorob'ev V S and Malyshenko S P $2004 J$. Phys. D: Appl. Phys. 37495 